\title{
Investigation of phase-equivalent potentials by a halo transfer reaction
}

\author{
B. Gönül; O. Özer and M. Yilmaz \\ Department of Engineering Physics, University of Gaziantep, \\ 27310 Gaziantep-Türkiye
}

\begin{abstract}
Using the supersymmetric quantum mechanics we investigate the wave function-sensitive properties of the supersymmetric potentials which have received a lot of attention in the literature recently. We show that a superdeep potential and its phase-equivalent shallow-partner potential give very similar $r m s$ values for the weakly bound systems such as the deuteron and ${ }^{11} \mathrm{Be}$ nuclei. Although the corresponding eigenstates differ in the nodenumber, our investigation on the ${ }^{11} \mathrm{Be}(p, d){ }^{10} \mathrm{Be}$ single nucleon halo transfer reaction at $35 \mathrm{MeV}$ show that also other physical quantities such as the cross section angular distributions calculated using these wave functions reflect the nodal structure rather weakly. This lends support to two nearly equivalent treatments of the Pauli principle.
\end{abstract}

Published in Eur. Phys. J. A 9, 19-28 (2000)

\section{Introduction}

The long-standing dichotomy of choosing a shallow or deep effective local potential to describe nucleus-nucleus elastic scattering was greatly clarified by Baye [1], who demonstrated that in scattering, for all practical purposes, these two kinds of potentials in the literature [2], [3] are phase-equivalent supersymmetric partner potentials of each other. Supersymmetric quantum mechanics allows one to transform a hamiltonian to its partner such that they possess identical spectra or differ at most from each other by having the lowest eigenstate eliminated in its partner's spectrum 四. Repeated application of the supersymmetric transform could yield a hamiltonian which has a prescribed number of eigenstates less than the starting hamiltonian. By the same token, a supersymmetric transform can also add an eigenstate of the desired energy to the starting spectrum. The supersymmetric transform, apart from eliminating the lowest eigenstate, induces in the first instance a change in the phase shifts of the continuum states 
of the starting spectrum. Baye [1] showed that by a judicious choice of repeated supersymmetric transforms, the partner hamiltonian can be made to be phaseequivalent. Thus, in the case of $\alpha-\alpha$ scattering, where the starting hamiltonian has a deep effective local potential [3] known to possess bound states which are unphysical because of the Pauli principle, Baye eliminated these unphysical states by successive supersymmetric transform while preserving the phase equivalence. In the course of this process, the original potential is transformed to be singular and progressively shallower. Moreover, the supersymmetric transforms are performed for each angular momentum $(\ell)$ separately. Therefore, at the final stage, a set of shallow, energy-independent and $\ell$-dependent effective local potentials is obtained which is completely phase-equivalent to the original deep, energyindependent and $\ell$-independent potential. The set of shallow potentials bears a remarkable resemblance to the $\ell$-dependent $\alpha-\alpha$ potentials documented in the literature [2].

Such ambiguous choice in the nature of the potential to describe nucleusnucleus elastic scattering is found not only in $\alpha-\alpha$ but also in many other systems [5]. This dual picture of the nucleus-nucleus interaction arises most probably because the many-body description of the system has been simplified in different ways to a two-body interaction between two structureless particles. For the analysis of elastic scattering, such drastic difference in the character of the potentials is immaterial since only phase shifts are required and these potentials are phase-shift equivalent or in the main so. However, when one has to choose one of these potentials to be used in nuclear structure studies in which wave functions are explicitly involved, the implication of a deep or shallow potential may be immense. The supersymmetric procedure produces two sets of wave functions for weakly bound systems, which coincide at large distances but differ at small distances by the additional node appearing inside the core by use of the deep potential. Therefore, it is important to have a quantitative criteria to discriminate which potential has the correct wave function-sensitive properties.

These deep and shallow potentials were used independently by Baye et al. and Liu [6] to calculate bremsstrahlung emission in possible nucleus-nucleus collisions. The calculations seemed to indicate that while the bremsstrahlung cross sections from resonating-group method and the deep potential resemble each other, those of the shallow potential are distinctly different. Hence it was concluded that the deep potential is to be preferred over its shallow partner if wave function-sensitive properties are important.

Recently Dijk et al. [7], and Ridikas and his co-workers [7], have separately shown that a superdeep potential and its supersymmetric partner give very similar rms values for the model deuteron, and one-neutron halo systems (considering ${ }^{11} \mathrm{Be}$ nucleus), respectively. In the work of Ridikas et al. it was also stated that other physical quantities, which are more sensitive to the behavior of the radial wavefunctions in the nuclear interior, such as transition probabilities obtained by the deep potential and its phase equivalent partner, reflect the nodal structure 
rather weakly.

In order to complete our discussion, we repeat these calculations with some extent, considering a transfer reaction, ${ }^{11} \mathrm{Be}(p, d)^{10} \mathrm{Be}$, involving the weakly bound deuteron and single nucleon-halo ${ }^{11} B e$ nuclei which are well suited for studying the consequence of different wave functions from the deep and reconstructed phase equivalent shallow potentials on reaction observables. Current experimental activity in the area of light-neutron rich and drip-line nuclei now dictates the rapid development of calculable theoretical models for reactions and scattering of effective few-body systems. Hence, there is an increasing general interest in supersymmetric potentials in this context. Our results are contributions to the discussion in this subject -an investigation in a relatively unexplored area of the quantitative consequence of the supersymmetric potentials.

We begin with a brief sketch of the general method in section 2, where we also introduce a two-parameter, deep, shape invariant two-body potential used throughout the present calculations. In sections 3 and 4 , we discuss the application of the method to the deuteron and ${ }^{11} \mathrm{Be}$ nuclei respectively, giving the characteristic properties of the constructed phase-equivalent two-body potentials, and the connection between the exclusion of deep-lying Pauli forbidden bound states from some potential and supersymmetry is reviewed in the light of the calculation results. Section 5 discusses the ${ }^{11} B e(p, d)^{10} B e$ reaction calculations in terms of the supersymmetric partner potentials. Finally section 6 contains a summary and the conclusion.

\section{Supersymmetric quantum mechanics}

Supersymmetric quantum mechanics [8] and its connection to the factorization method [9] have been extensively investigated [10]. Since the ground state wave function $\Psi^{(n=0)}$ for a bound system has no nodes, it can be written as

$$
\Psi^{(n=0)}(r)=\exp \left(-\frac{\sqrt{2 \mu}}{\hbar} \int W(r) d r\right)
$$

Introducing the operators

$$
\widehat{B}=W(r)+\frac{i}{\sqrt{2 \mu}} \hat{p}, \widehat{B}^{+}=W(r)-\frac{i}{\sqrt{2 \mu}} \hat{p}
$$

the hamiltonian can be easily factorized

$$
\hat{H}-E^{(n=0)}=\widehat{B}^{+} \widehat{B}
$$

where $E^{(n=0)}$ is the ground state energy. Since the ground state wave function satisfies the condition

$$
\widehat{B}\left|\Psi^{(n=0)}\right\rangle=0
$$


the supersymmetric partner hamiltonians $\left(H_{m}, m=1,2, \ldots\right)$

$$
\hat{H}_{1}=\widehat{B}^{+} \widehat{B} \quad, \quad \hat{H}_{2}=\widehat{B} \widehat{B}^{+}
$$

have the same energy spectra except the ground state of $\hat{H}_{1}$, which has no corresponding state in the spectra of $H$. The corresponding supersymmetric partner potentials are given by

$$
V_{1}(r)=[W(r)]^{2}-\frac{\hbar}{\sqrt{2 \mu}} \frac{d W}{d r}, V_{2}(r)=[W(r)]^{2}+\frac{\hbar}{\sqrt{2 \mu}} \frac{d W}{d r}
$$

It was shown that a subset of the potentials for which the Schrödinger equations are exactly solvable share an integrability condition called shape invariance [11]. The partner potentials of Eq. (6) are called shape invariant if they satisfy the condition

$$
V_{2}\left(r ; a_{1}\right)=V_{1}\left(r ; a_{2}\right)+R\left(a_{1}\right)
$$

where $a_{1,2}$ are a set of parameters that specify space-independent properties of the potentials (such as strength, range, and diffuseness), $a_{2}$ is a function of $a_{1}$, and the remainder $R\left(a_{1}\right)$ is independent of $r$.

An iterative procedure within the supersymmetric quantum mechanics framework for building the partner of a given potential admitting the same eigenvalues except for that of the missing ground state was proposed by Baye [1] on the basis of a general procedure due to Sukumar [4], [12]. The method relies on a factorization property of the hamiltonian, and makes possible the (exact) construction of the partner potential starting from the original potential ground state wave function. It actually requires two steps, the intermediate potential $\left(V_{2}\right)$ having the same negative energy spectrum for a bound system as the original potential $\left(V_{1}\right)$, except for the ground state of the latter, but a different phase shift; $V_{2}(r)$ is given by

$$
V_{2}(r)=V_{1}(r)-2 \frac{\hbar^{2}}{2 \mu} \frac{d^{2}}{d r^{2}} \ln \Psi_{1}
$$

where $\Psi_{1}\left(E_{1}^{(n=0)}\right)$ denotes the original ground state wave function. The second step provides $V_{3}(r)$ the final phase-equivalent potential (PEP) as

$$
V_{3}(r)=V_{1}(r)-2 \frac{\hbar^{2}}{2 \mu} \frac{d^{2}}{d r^{2}} \ln \left[\Psi_{1}\left(E_{1}^{(n=0)}\right) \Psi_{2}\left(E_{1}^{(n=0)}\right)\right]
$$

where $\Psi_{2}\left(E_{1}^{(n=0)}\right)$ stands for the wave function at the same energy, but calculated with the intermediate potential $V_{2}$. Eq. (9) can also be reduced to the form

$$
V_{3}(r)=V_{1}(r)-2 \frac{\hbar^{2}}{2 \mu} \frac{d^{2}}{d r^{2}}\left\{\ln \int_{0}^{r}\left[\Psi_{1}\left(E_{1}^{(n=0)}, r^{\prime}\right)\right]^{2} d r^{\prime}\right\}
$$

Elimination of more than one state is accomplished by iterating this two-step procedure. 


\subsection{A two-parameter superdeep potential}

In Ref.[13], the on-shell equivalence of the deep quantum-chromodynamically motivated realistic nucleon-nucleon interaction proposed by Kukulin et al. [14 with more conventional repulsive-core forces has been investigated by eliminating its unphysical deeply bound states, while preserving its scattering properties and the binding energy of the deuteron. Using the spirit of this work, and of Ref. [7], here we use an alternative superdeep potential. As a simple, physically interesting example, consider the potential

$$
V(r)=-V_{0} \operatorname{sech}^{2} \beta r
$$

Potentials of this shape can be generated from the superpotential [15],

$$
W(r)=A \tanh \beta r, \quad A>0 .
$$

In fact, using Eq. (6), the supersymmetric partner potentials are

$$
\begin{aligned}
& V_{1}(r ; A)=A^{2}-A\left(A+\frac{\beta \hbar}{\sqrt{2 \mu}}\right) \operatorname{sech}^{2} \beta r, \\
& V_{2}(r ; A)=A^{2}-A\left(A-\frac{\beta \hbar}{\sqrt{2 \mu}}\right) \operatorname{sech}^{2} \beta r .
\end{aligned}
$$

Clearly, one can write

$$
V_{2}(r ; A)=V_{1}\left(r ; A-\frac{\beta \hbar}{\sqrt{2 \mu}}\right)+A^{2}-A\left(A-\frac{\beta \hbar}{\sqrt{2 \mu}}\right)^{2},
$$

which is precisely the requirement of Eq. (7) for shape invariance. Therefore the bound state energies of the potential $V_{1}$ are

$$
E_{1}^{(n)}=A^{2}-\left(A-n \frac{\beta \hbar}{\sqrt{2 \mu}}\right)^{2}
$$

The energy levels $E^{(n)}$ of the original potential given by Eq. (11) can be obtained by subtracting $A^{2}$ from $E_{1}^{(n)}$ and identifying

$$
V_{0}=A\left(A+\frac{\beta \hbar}{\sqrt{2 \mu}}\right)
$$

Solving for $A$ and requiring $A>0$ gives

$$
A=-\frac{\beta \hbar}{2 \sqrt{2 \mu}}+\frac{1}{2} \sqrt{\frac{\beta^{2} \hbar^{2}}{\sqrt{2 \mu}}+4 V_{0}} .
$$


Therefore, the energy levels of the deep potential $V(r)=-V_{0} \operatorname{sech}^{2} \beta r$ are

$$
E^{(n)}=E_{1}^{(n)}-A^{2}=-\left(A-\frac{n \beta \hbar}{\sqrt{2 \mu}}\right)^{2},
$$

which is well known to be the correct answer [16]. As we deal with the bound systems, we require the odd solutions due to boundary conditions. Hence replacing $n$ in Eq. (18) by $2 n+1$ term, we arrive at ,

$$
E^{(n)}=-\frac{\hbar^{2}}{2 \mu}(\tilde{A}-2 n-1)^{2} \beta^{2}, \quad n=0,1,2, \ldots,
$$

where $\tilde{A}=\frac{A}{(\hbar \beta / \sqrt{2 \mu})}$. The depth of the potential given by Eq. (16) reduces in this case to the form

$$
\tilde{V}_{0}=-\frac{\hbar^{2}}{2 \mu} \tilde{A}(\tilde{A}+1) \beta^{2}
$$

Eqs. (19) and (20) are in consistent with the expressions used in Ref. [7] where also the analytical expressions for the wave functions of the ground and first excited state corresponding the potential of interest can be found.

We have first employed this deep sech-squared potential in analyzing the alpha-alpha scattering (but not discussed here) by choosing the two parameters as $\tilde{A}=5.945$ and $\beta=0.535 \mathrm{fm}^{-1}$, together with $\frac{\hbar^{2}}{2 \mu_{\alpha-\alpha}}=10.375 \mathrm{MeV} \mathrm{fm}^{2}$. We have observed that $-V_{0} \operatorname{sech}^{2} \beta r \rightarrow-U_{0} \exp ^{\left(-\alpha r^{2}\right)}$ with $U_{0}=122.694 \mathrm{MeV}$, $\alpha=0.22 \mathrm{fm}^{-2}$ and have reproduced successfully the Figs. (1,2) of Ref. [1] using this two-parameter shape invariant superdeep potential, without involving a gaussian type potential in the calculations. However, as we deal with ${ }^{11} B e(p, d){ }^{10} B e$ reaction calculations throughout the present work, we focus on the treatment of deuteron and ${ }^{11} \mathrm{Be}$ ground state wave functions in the supersymmetric quantum mechanical framework.

\section{Application to the deuteron system}

All the available "realistic" nucleon-nucleon forces are characterized by a relatively weak central attractive part and by the presence of a hard or soft repulsive core at small distances; the first feature reflects the loose binding of the neutronproton system, while the introduction of a repulsive core is required by the negative values assumed by the low-experimental phase-shifts when energy increases. However the feasibility of a description of comparable quality of the low energy properties of the two-nucleon system in the ${ }^{1} S_{0}$ and ${ }^{3} S_{1}-{ }^{3} D_{1}$ channels (including deuteron properties), in terms of a deep, purely attractive interaction called Moscow potential was demonstrated by Kukulin and his co-workers [14]. Their potential differs from those obtained in the more traditional approaches by the 
existence of an additional deeply-bound state in each channel, and by an increase of the absolute singlet and triplet phase-shifts due to this extra unphysical bound state. It is well known from cluster nuclear physics that these seemingly contradictory features - that is, repulsive core versus deep potential descriptions - are two ways to simulate the effects of the Pauli principle in a local potential model description when the two interacting particles are composed of identical fermions. There have been quite a few attempts to derive the features of the two-nucleon interaction from a quark picture of the nucleon. The most non-relativistic quark model calculations led to an effective nucleon-nucleon interaction with a strong repulsive core and an intermediate range attraction similar to those displayed by the empirical potentials. On the other hand, the work described in Ref.[14] indicated, for the nucleon-nucleon scattering, that the relative s-wave function has to have a node at small distance. The existence of a node in the relative motion wave function can readily incorporated in a local potential description, provided interaction is deep enough to accommodate one (nodeless) deeply bound state, such as the one proposed by Kukulin and his co-workers. The work described here will, in addition to the other investigations undertaken, demonstrate explicitly the equivalence of such deep potentials with the more orthodox repulsive core empirical interactions, by constructing the phase-equivalent supersymmetric partner of the deep potential freed from unphysical bound states but still binding the deuteron with correct energy. The resulting supersymmetric potential for the deuteron case are to be shown to have a short range repulsive core followed by a shallow attractive part, which are very similar to those displayed by realistic interactions such as the Reid soft core potential [17].

\subsection{Phase-equivalent potentials for the deuteron}

Now, superdeep potentials such as the Moscow potential give deuteron wave functions with a node at short distances. The node arises because there is an additional bound state which is Pauli forbidden for the actual neutron-proton $(n-p)$ system. The latest version of Moscow potential [14 includes both central and a tensor component, together with the central and tensor one-pion exchange contributions (OPEP). In addition, the equations in Ref.[14] to solve both for the bound state ( $S$ - and $D$-wave) and the scattering problem in case of triplet potential are more complicated, one needs to work out the usual coupled equations. For simplicity, in present calculations we use an alternative superdeep potential discussed in Section 2.1, which produces a model deuteron wave function that has a node like the Moscow potential. However, this simple potential does not have the required OPEP tail and do not consider $D$-wave related to the tensor potential. So the physical observables calculated by this potential, such as the radius of the deuteron which we will deal with later in this section, should not be compared with the experimental value. We note at this point that our aim here is not the rigorous reproduction of experimental data but to test the reli- 
ability of a deep potential description involving some unphysical bound states, and in particular to search for the wave function sensitivity features of the deep and of its supersymmetric partner-shallow potentials used both in the entrance and exit channels of the ${ }^{11} \mathrm{Be}(p, d){ }^{10} \mathrm{Be}$ reaction. For this reason, the use of an appropriate simple potential, such as the binary sech-squared potential, in the present analysis does not cause any physical problem.

Considering the well-known charge radius formula

$$
R^{2}(\operatorname{charge})=\frac{1}{2} R_{p}^{2}+\frac{1}{4} R_{r m s}^{2}
$$

with $R_{p}$ being the proton radius and $R_{r m s}$ the mean-square intercluster distance (matter radius), one can determine the free parameters $\tilde{A}$ and $\beta$ for the potential considered in analyzing the deuteron nucleus by solving the following system equations ;

$$
\begin{aligned}
E^{(n)} & =-\frac{\hbar^{2}}{2 \mu_{n-p}}(\tilde{A}-2 n-1)=-2.226 \mathrm{MeV}, \\
R_{r m s}^{2} & =\frac{1}{4} \int d r r^{2} \Psi_{m}^{(n)}(\tilde{A}, \beta, r)^{2}=\left(\begin{array}{ll}
1.95 \mathrm{fm}
\end{array}\right),
\end{aligned}
$$

where $n$ denotes the energy level as stated earlier and $m$ refers to $m^{\text {th }}$ hamiltonian. Throughout our calculations, $\frac{\hbar^{2}}{2 \mu_{n-p}}$ is set $41.47 \mathrm{MeV} \mathrm{fm}^{2}$ and the arbitrary constants $\tilde{A}$ and $\beta$, for the Moscow-type binary potential, are calculated as 3.146 and $1.587 \mathrm{fm}^{-1}$ respectively. In this case, from Eq. (22), the ground state has a binding energy of about $481 \mathrm{MeV}$, which is unphysical and needs to be suppressed. The physically meaningful deuteron bound state for this superdeep potential corresponds to the first excited state having a binding energy of 2.226 $\mathrm{MeV}$.

The building the partner of a given potential admitting the same eigenvalues except for that of the missing ground state has been discussed in Section 2. The two-parameter superdeep potential- $V_{1}(r)$ and its phase-equivalent supersymmetric partner- $V_{3}(r)$, together with the intermediate non-phase-equivalent potential $V_{2}(r)$, are shown in Fig. 1-a, and their corresponding wave functions in Fig. 2. As a result of the presence of one spurious bound state, the deuteron wave function for the superdeep potential possesses one node near the origin (around $0.56 \mathrm{fm}$ ). Figure $1 \mathrm{~b}$ compares the phase equivalent repulsive core interaction $\left(V_{3}\right)$ with the central part of the usual realistic Reid soft core potential [17]. The general futures of the PEP (such as the radius of the repulsive core and the strength of the attractive part) are seen to be similar to those of the Reid soft core potential. In spite of different analytical behavior near the origin for both Reid Soft core interaction (behaves at $r \rightarrow 0$ as $e^{-(\operatorname{const} \times x)} / x$ ) and the transformed phase equivalent shallow potential $\left(V_{3}(r)_{r \rightarrow 0} \approx\left(\right.\right.$ const $\left.\left./ r^{2}\right)\right)$, we observe in the figure a considerable similarity of both interactions. This means we have very tight 
interrelation between a deep nucleon-nucleon model potential and the standard Reid soft core interaction, which will be discussed later in this section.

It is seen from fig. 2 that our reconstructed phase-equivalent supersymmetric partner potential $\left(V_{3}\right)$ has led to relative motion wave functions very similar to this generated by the deep potential $\left(V_{1}\right)$ outside the core region, but which lack the small distance radial node resulting from the suppression of the unphysical bound state. If there is a node in the wave function and the wave function is reasonably large at small distances, then one might expect that because of the normalization the wave function at large distances would be reduced. In other words, the asymptotic phase-equivalent wave function will have a smaller value of the asymptotic amplitude and hence the radius would be reduced. To clarify that if these wave functions having different behavior inside the core lead to quantitatively different results, we investigate the dependence of radius calculations, as an observable, on the wave function properties.

The deuteron matter radius $R_{r m s}$ can be calculated numerically from Eq. (22) for either $\Psi(r)=\Psi_{1}^{(n=1)}(r)$ or $\Psi(r)=\Psi_{3}^{(n=0)}(r)$, the bound state wave functions for the superdeep and transformed partner potentials. The numerical calculations of $r m s$ value (root-mean-square radius) show that the radius of deuteron is 1.953 $\mathrm{fm}$ and $1.955 \mathrm{fm}$ for the superdeep potential and PEP respectively, which are so close. That means as a physical quantity, the radius calculated using the wave functions having one-/no-node reflect the nodal structure rather weakly. But, in case of experiment requiring a reduction in $R_{r m s}$ for a nucleus then short range contribution to the potential appears, such as the superdeep potential used here, would be necessary.

In order to check the accuracy of the supersymmetric quantum mechanical methods used in constructing the phase-equivalent potential, we have carried out additional calculations on the phase-shift. The resulting phase-shift curve obtained by PEP $\left(V_{3}(r)\right)$ is compared with that obtained by the deep $\left(V_{1}\right)$ potential and an excellent agreement between the resulting and initial phase-shifts is observed.

To summarize, when nucleons are endowed with quark structures, nucleonnucleon bound and scattering properties can be described by a deep potential $(\approx 1000 \mathrm{MeV})$, whose supersymmetric partner potential is singular and looks teasingly like the Reid soft-core or similar shallow potentials. One can make a conclusion at this stage on the supersymmetry aspect of nucleon-nucleon interaction in the light of the calculation results obtained, making a possible connection between the exclusion of deep-lying bound states from some potential and supersymmetry. This very interesting aspect is connected with very deep interrelation between many-body and potential treatment for the composite particle interaction in case of the particles composed of elementary fermions. From physical point of view this interrelation means the existence of a deeply hidden relation between the relative motion of composite nucleus comprised from fermions and 
the internal excitations of the composites. In fact, in non-relativistic quantum mechanics which is used for the treatment of interaction of the composite particles, the relative motion of the composites is treated as a bosonic degree of freedom (i.e., no Pauli principle constrains are put to the relative motion). On the other hand, the internal excitations of the quark degrees of freedom inside the composites in the process of the mutual collision of the composites should be treated as the manifestation of fermionic degrees of freedom. The main problem in description of composite particle interaction is the complicated interrelation between relative motion of the composites and their inner excitations. And from this point of view, the existence of the above supersymmetry aspect could mean that the collision of such composites should be described correctly only within the framework of supersymmetrical quantum mechanics and is only the projection of this nontrivial picture onto mutual relative motion of the composites.

As a conclusion, by means of supersymmetric quantum mechanics we have found that the two-parameter superdeep potential and its supersymmetric partner, which is phase-equivalent to the former and freed from the unphysical deeply bound states, give very similar rms values for the deuteron system. Although the corresponding eigenstates differ in the node-number, our investigations have shown that the matter radius calculations using these wave functions reflect the nodal structure rather weakly. This lends support to two nearly equivalent treatments of the Pauli principle by choosing the physical solution either by nodenumber criteria or by inclusion of a repulsive part of the potential at the origin. However, the similar rms results obtained do not automatically imply that other observables such us differential cross section, vector analyzing power calculated using these wave functions have to coincide. Clearly considerable additional work is still needed to test further the virtues of a deep potential description of the nucleon-nucleon interaction, which will be discussed in detail in Section 5 considering a halo transfer reaction. However, as the ${ }^{11} \mathrm{Be}$ nucleus in the entrance channel of the reaction considered is weakly bound, like the deuteron in the exit channel, a similar discussion for the ${ }^{11} \mathrm{Be}$ system being one-neutron halo nucleus within the framework of supersymmetric quantum mechanics is necessary before proceeding.

\section{Application to the ${ }^{11} B e$ system}

Research with radioactive nuclear beams is currently one of the most active areas in nuclear physics. As one of the successful applications of such nuclear beams, exotic structures have been observed in nuclei near to the driplines, which are called, as the most interesting discoveries, the neutron halo. These nuclei have opened studies of weakly bound nuclear systems, which has not freely accessible before. These nuclei, such as ${ }^{11} \mathrm{Be}$, have long-range wave functions and are characterized by a cloud, or halo, of neutron probability that extends far beyond 
the dense core. According to classical physics such nuclei should not exist at all because the strong nuclear force (the glue that binds neutrons and protons together) has too short to hold the far off neutrons in the halo. Instead, they owe their existence to quantum theory which describes the location of subatomic particles by a mathematical cloud of probability. In [18 and references therein essential features of loosely bound systems, having an unusually large size, are discussed. In this Section, as an example to halo systems, we examine the ground state of ${ }^{11} B e$, which consists of a single neutron halo with a ${ }^{10} B e$ core nucleus, using the supersymmetric quantum mechanics. It is well known that the dominant component of the ${ }^{11} \mathrm{Be}$ ground state is produced by the coupling of a $1 s_{1 / 2}$ neutron, having the separation energy of $0.503 \mathrm{MeV}$, to a ${ }^{10} \mathrm{Be}$ core.

\subsection{Phase-equivalent potentials for the ${ }^{11} B e$ system}

In this analysis, which will be restricted to the $s$-motion only, we again make use of the sech-squared deep potential with appropriate physical parameters. At this stage we should stress that one can relate the matter root-mean-square radius $R_{R M S}$ (matter) to the single neutron root-mean-square radius $R_{r m s}$ by the formula [18]

$$
R_{R M S}^{2}(\text { matter })=\frac{W}{(W+1)} R_{R M S}^{2}(\text { core })+\frac{W}{(W+1)^{2}} R_{r m s}^{2}
$$

where $W$ is the mass number of the core, here the ${ }^{10} B e$ nucleus. Using [7] the $R_{R M S}($ core $)=2.3 \mathrm{fm}$ and $R_{R M S}($ matter $)=2.73 \mathrm{fm}$, it is easy to check that the value $R_{r m s}=6.70 \mathrm{fm}$ gives roughly the average of the measured values of $R_{R M S}($ matter $)$.

To determine the two free parameters $(\tilde{A}, \beta)$, one needs to solve the following equations leading to the correct rms value and binding energy for the $B e-11$ system,

$$
\begin{aligned}
E^{(n)} & =-\frac{\hbar^{2}}{2 \mu}(\tilde{A}-2 n-1)=-0.503 \mathrm{MeV} ; \\
R_{r m s}^{2} & =\int d r r^{2} \Psi_{m}^{(n)}(\tilde{A}, \beta, r)^{2}=(6.70 \mathrm{fm})
\end{aligned}
$$

where $\mu$ is the reduced mass of the system: ${ }^{10} \mathrm{Be}+n$.

The physical solution is chosen using the node-number and parity quantum number criteria. As we take into account that $0 s_{1 / 2}$ the orbit is completely occupied and put the single neutron in $1 s_{1 / 2}$ state (having one node in the wave function) to have a positive parity required by the experiment, we choose the excited state wave function with one node, instead of ground state, as a physically meaningful solution for the superdeep potential in analyzing the ground state of ${ }^{11} \mathrm{Be}$ nucleus. 
Now we are ready to apply the supersymmetric technique to create the PEP corresponding to the sech-squared superdeep potential for the ${ }^{11} \mathrm{Be}$ one-nucleon halo system, with the calculated values of $\tilde{A}=3.124$, and $\beta=0.694 \mathrm{fm}^{-1}$ together with $\frac{\hbar^{2}}{2 \mu}=22.81 \mathrm{MeV} \mathrm{fm}^{2}$. Using the formulae given by the previous sections, the supersymmetric partner potentials and corresponding eigenstates are calculated. Fig. 3 illustrates the superdeep and related partner potentials while Fig. 4 gives their eigenstates respectively. It is seen from Fig. 4 that the eigenfunction corresponding to the PEP is a nodeless ground state. In this case the Pauli principle is taken into account by the repulsive part of the potential, see Fig. 3, repulsive up to $1.5 \mathrm{fm}$ approximately.

If one calculates the $R_{r m s}$ for the system of interest using the nodeless eigenstate of PEP, the value of $6.78 \mathrm{fm}$ is obtained. Even the wave functions differ in the node number, like the deuteron case discussed in Section 3, the $R_{r m s}$ is nearly the same for the phase-equivalent potentials, which is found about 6.70 $\mathrm{fm}$ for the initial deep potential, whereas $6.17 \mathrm{fm}$ for the intermediate nonphase-equivalent potential. One should not forget that the non-PEP potential does not belong to the PEP family, that is why the corresponding $R_{r m s}$ values so close for the PEP potentials while that of non-PEP considerably differ than the others. Moreover, the corresponding eigenfunction of this non-PEP potential has a different asymptotic behavior as well.

We have also calculated the $s$-wave phase shifts in case of ${ }^{10} B e(d, p)^{11} B e$ elastic scattering up to $20 \mathrm{MeV}$ for the three potentials. The results clearly exhibit the difference between PEP and non-PEP.

\section{Application to the ${ }^{11} B e(p, d){ }^{10} B e$ reaction at $35 \mathrm{MeV}$}

Single nucleon transfer reactions, such as the $(d, p)$ and $(p, d)$ reactions, have been a reliable tool in nuclear spectroscopic studies of stable nuclei, determining positions, spins and parities of nuclear states. Recently, the use of low energy single nucleon transfer reactions for structure studies of exotic nuclei have attracted attention [19]-20]. Because of the simplicity of the theoretical interpretation of these reactions, they are thought to provide an important source of the information about the structure of halo nuclei, such as ${ }^{11} B e$. It is now understood that the $1 s_{1 / 2}$ neutron single particle state in this region is lowered and that a dominant component of the ${ }^{11} \mathrm{Be}$ ground state is produced by the coupling of a $1 s_{1 / 2}$ neutron to a ${ }^{10} \mathrm{Be}$ (g.s., $0^{+}$) core; with a smaller but significant component in which a $0 d_{5 / 2}$ neutron is coupled to a $2^{+}$excitation of the ${ }^{10} \mathrm{Be}$ core.

The importance upon such transfer reaction spectroscopic studies of the inclusion of the deuteron breakup degrees of freedom has been well discussed in Ref. [20] via the theories used to analyze measured cross section observables, and 
shown that the magnitudes of the calculated cross sections, and particularly the ratio of the cross sections to the ground state and $2^{+}$core states, of ${ }^{10} \mathrm{Be}$ are affected by the inclusion of three-body channels.

Here in this section we do not discuss the details of these calculations. The aim of the present calculations is to investigate how the calculated physical observables of the reaction sensitive to the nodal structure, involving the deuteron and bound neutron wave functions discussed in the previous sections with one node/no node.

We calculate the transfer amplitude using the prior form of the $(p, d)$ matrix element, thus the transition interaction is the $n-p$ interaction and we need a full (three-body) description of the $n+p+{ }^{10} B e$ system in the final state. For the description of this final state we have used both the adiabatic (AD) model [21] and the quasi-adiabatic (QAD) approach [22]. However, for the sake of clarity in discussing the physics behind this application, we here consider only the AD model calculations as both model calculation results have led us to the same conclusion.

In the context of $(p, d)$ reactions the outgoing deuteron wave function, $\Psi_{d}$, enters the transition amplitude

$$
T_{p d}=\left\langle\Psi_{d}(\bar{r}, \bar{R})\left|V_{n p}\right| \chi_{p}\left(\bar{r}_{p}\right) \phi_{n}\left(\bar{r}_{n}\right)\right\rangle
$$

where $\bar{r}\left(=\bar{r}_{p}-\bar{r}_{n}\right)$ is the relative coordinate of the $n-p$ pair and $\bar{R}\left[=\frac{1}{2}\left(\bar{r}_{p}+\bar{r}_{n}\right)\right]$ is the center of mass coordinate. Here $\phi_{n}$ is the neutron bound state and $\chi_{p}$ incoming proton wave function. The vectors $\bar{r}_{n}$ and $\bar{r}_{p}$ are the positions of the transferred neutron with respect to the ${ }^{10} \mathrm{Be}$ core and of the proton relative to the ${ }^{11} \mathrm{Be}$ system. The cross section (in the center of mass frame) for neutron pickup to bound $n-p$ pair is then given by

$$
\frac{d \sigma_{p d}}{d \Omega_{f}}=\frac{\mu_{i} \mu_{f}}{\left(2 \pi \hbar^{2}\right)^{2}} \frac{k_{f}}{k_{i}}\left|T_{p d}\right|^{2}
$$

where $d \Omega_{f}$ is the element of the solid angle for the asymptotic center of mass momentum of the bound deuteron. In the above $\mu_{i}$ and $\mu_{f}$ are the center of mass relative motion reduced masses in the initial and final channels, $k_{i}$ and $k_{f}$ are the entrance and exit channel wave numbers, respectively. Throughout this paper we restrict the formalism to $s$-wave $n-p$ relative motion for simplicity. In zero-range

approximation then it is the wave function at coincidence, $\Psi_{d}(r \approx 0, \bar{R})$ which is of importance.

\subsection{Transition amplitudes in zero-range approximation}

It is important for the present work to make clear the essential differences in the calculations carried out using both phase-equivalent potentials, the initial 
superdeep sech-squared potential and the shallow phase-equivalent partner potential, in calculating the ground state deuteron wave function in the final state and for the calculation of the transferred neutron bound state wave function in the initial channel of the reaction. So they are developed in some detail within a common notation. The calculations employed the deep potential (denoted by $V_{1}$ in sections through $2-4$ ) will be represented by the script deep in the following formulae and for the phase-equivalent shallow potential (represented by $V_{3}$ earlier) calculations we use the script pep.

The transition amplitudes for the processes are evaluated in a zero-range approximation, and the related relative amplitudes, of primary interest here, are accurately described. For clarity we will not show the transferred neutron spectroscopic factor or any spin projection labels explicitly.

In the adiabatic approximation the required transition amplitudes are

$$
T_{p d}^{\text {deep }(\text { pep })}=\left\langle\chi_{d}(r, \bar{R}) \Phi_{d}^{\text {deep }(p e p)}(r)\left|V_{n p}^{\text {deep }(p e p)}(r)\right| \chi_{p}\left(\bar{r}_{p}\right) \phi_{n}^{\text {deep }(p e p)}\left(\bar{r}_{n}\right)\right\rangle
$$

As in calculating the transfer amplitudes, we make use of the zero-range approximation, thus for the bound deuteron we replace

$$
V_{n p}^{\text {deep }(p e p)}(r) \chi_{d}(r, \bar{R}) \phi_{d}^{\text {deep }(p e p)}(r) \approx D_{0}^{\text {deep }(p e p)}(p, d) \chi_{d}(r \approx 0, \bar{R}) \delta(r)
$$

with strength parameters

$$
D_{0}^{\text {deep }(p e p)}(p, d)=\sqrt{4 \pi} \int d r r V_{n p}^{\text {deep }(p e p)}(r) u_{0}^{\text {deep }(p e p)}(r),
$$

where $u_{0}$ is the radial deuteron ground state wave function $\left(\ell_{n p}=0\right)$ such that $\Phi_{d}(r)=\frac{1}{\sqrt{4 \pi}} \frac{u_{0}(r)}{r}$. The similar replacement should also be done for the QAD calculations.

It follows that,

$$
\begin{aligned}
T_{p d}^{\text {deep }(\text { pep })} & =D_{0}^{\text {deep }(p e p)}(p, d) M^{\text {deep }(p e p)}(p, d) \\
& =D_{0}^{\text {deep }(p e p)}(p, d)\left\langle\chi_{d}(r \approx 0, \bar{R}) \mid \chi_{p}(\gamma \bar{R}) \phi_{n}^{\text {deep }(p e p)}(\bar{R})\right\rangle
\end{aligned}
$$

where $\gamma=\frac{W}{W+1}$. Finally, considering the general expression for the cross section, we obtain

$$
\frac{d \sigma_{p d}^{\text {deep }(\text { pep })}}{d \Omega_{f}}=\frac{\mu_{i} \mu_{f}}{\left(2 \pi \hbar^{2}\right)^{2}} \frac{k_{f}}{k_{i}} D_{0}^{2}(\text { deep }, \text { pep })\left|M^{\text {deep }(\text { pep })}(p, d)\right|^{2}
$$

for the calculations used the adiabatic model in describing the final state. 


\subsection{Calculation methods}

We calculate the cross section angular distributions for the ${ }^{11} B e(p, d)^{10} B e$ single nucleon transfer reaction leading to the $0^{+}$ground state $\left(1 s_{1 / 2}\right.$ neutron transfer) of ${ }^{10} \mathrm{Be}$. We perform zero-range calculations using a modified version of the program TWOFNR [23]. The program has been further modified so that the calculated adiabatic and quasi-adiabatic radial wave functions, and appropriate zero-range strengths calculated for the phase equivalent deep and shallow potentials by Eq. (41), can be read in, together with the transferred neutron bound state $\phi_{n}$ obtained using the superdeep sech-squared potential and its phase-equivalent partner. In the three-body model calculations of the deuteron channel wave function $\left(d+{ }^{10} \mathrm{Be}\right)$, we make use of the global optical potential parameter set of Bechetti and Greenlees [24] evaluated at half the final state center of mass energy. The spin-orbit interactions are included. The entrance channel proton optical potential parameters are taken from [24]. The spin-orbit force in the proton channel is fixed at $6 \mathrm{MeV}$. The radial integrals are carried out from 0 to $35 \mathrm{fm}$ in steps of $0.1 \mathrm{fm}$. The maximum number of partial waves used was 30 for both the entrance and exit channels. The spectroscopic factors are set to unity throughout the calculations. All calculations presented here are done without non-locality corrections. Such corrections for halo transfer are expected to be small because they correct the transition amplitude in the nuclear interior, but the long tail of the halo wave function makes internal contributions less important.

\subsection{Results and discussion}

It is useful at this stage to remind ourselves again that the deep potential and its phase equivalent shallow partner, which are used for calculating the bound deuteron and transferred neutron wave functions, are constructed to have identical phase shifts so that any difference in the transition amplitudes, and in the cross section angular distributions, is attributed entirely to the corresponding wavefunctions of the partner potentials.

The result for the adiabatic model cross section angular distributions for the reaction involving the original deep potential description and its comparison with that obtained by means of the PEP description is given in Fig. 5. The figure indicates almost complete coincidence of both curves. This may be understood from the following analysis. The two-body supersymmetric partner potential dependence in the differential cross section calculations, originates from two terms: the zero-range constant $D_{0}^{2}$, and the transition amplitude $|M(p, d)|^{2}$ through the deuteron and bound neutron ground state wave functions. The calculated transfer strengths, for the deep potential description of the $n-p$ interaction is $D_{0}^{2}($ deep $)=15792 \mathrm{MeV}^{2} \mathrm{fm}^{3}$ while $D_{0}^{2}(\mathrm{pep})=15980 \mathrm{MeV}^{2} \mathrm{fm}^{3}$ for the shallow partner description. It is also worth stressing that, from the results obtained in sections 3 and 4, the transition from the deep potential to the repulsive core in- 
teraction (PEP) does not significantly alter the outer part of the neutron ground state wave functions appear in the transition amplitude. And in addition, the neutron halo wave function makes internal contribution less important. Therefore $\left|M^{\text {deep }}(p, d)\right|^{2} \approx\left|M^{p e p}(p, d)\right|^{2}$, and the ratio of the cross sections

$$
\begin{aligned}
\frac{d \sigma_{p d}^{\text {deep }}}{d \Omega_{p d}^{p e p}} & \approx \frac{D_{0}^{2}(\text { deep })}{D_{0}^{2}(\text { pep })}=\frac{15792 \mathrm{MeV}^{2} \mathrm{fm}^{3}}{15980 \mathrm{MeV}^{2} f m^{3}} \\
& =0.988 \approx 1
\end{aligned}
$$

which leads to the coincidence of the results.

\section{Conclusion}

The properties of the deep nuclear interaction have been investigated by constructing explicitly phase-equivalent potentials freed from the unphysical deeply bound states of the former. We have seen that the resulting central potentials have to be repulsive and singular at small distance in order to preserve the energy behavior of the phase shifts, and they present a shallow attractive part of intermediate range. Our reconstructed potentials (PEP) have led to relative motion wave functions very similar to those generated by the deep potentials outside the core region, but which lack the small distance radial node. Both types of potentials are therefore expected to display rather different off-shell behaviors, and presumably lead to qualitatively different results. However, there is no considerable discrepancy between the rms calculation results of these quite different two-body interaction descriptions has been found. Nevertheless, if experiment actually does require a reduction in observables then a short range non-local contribution to the potential, like the deep potential, appears to be necessary.

We have used phase equivalent two-body potentials with a different number of bound states considering the ${ }^{11} \mathrm{Be}(p, d){ }^{10} \mathrm{Be}$ reaction at $35 \mathrm{MeV}$, and compared the calculated corresponding physical observables. Investigation of the consequences of using these completely phase equivalent two-body potentials for the description of weakly bound deuteron and ${ }^{11} \mathrm{Be}$ nuclei in three-body calculations, based on the adiabatic approach has led us to almost indistinguishable results. Due to the large spatial extension of halos, involving the simplest halo nucleus the deuteron and ${ }^{11} \mathrm{Be}$ as a one-neutron halo system, the probability is by definition very small at small distances. Hence, we conclude that the short range behavior of the corresponding wavefunctions for the deep and phase-equivalent shallow potentials, which coincide at large distances but differ at small distances by the additional node appearing inside the core by use of the deep potential, is not significant for the analysis of such reactions.

In sum, the supersymmetric formulations used through the present calculations have dealt in general with the Pauli principle for the weakly bound systems. 
Assuming that the two-body potentials have Pauli forbidden states, one can then construct easily and use the phase equivalent partners without these forbidden states. At small distances the lowest levels of the original deep potentials correspond to identical fermions occupying the same states. Removal of these terms therefore forces the particles to occupy higher-lying orbits and thereby introducing the necessary repulsion preventing violation of the Pauli principle. In conclusion, this method to exclude the Pauli forbidden states in the weakly bound systems has firm mathematical and numerical foundations. It is a practical and accurate alternative to the other existing methods, such as the work described in Ref. 25] where an analytical $s$-wave potential with one bound state, which is the most important case in the practical applications for halo states, has been introduced. We note that the application of this potential, with the appropriate choice of the parameters involved, to the weakly bound deuteron and ${ }^{11} \mathrm{Be}$ nuclei has led to the similar results to those obtained by the shallow supersymmetric phase-equivalent partner potentials.

The valuable discussions with D. Baye, W. Van Dijk, and M. W. Kermode are here gratefully acknowledged. 


\section{References}

[1] D. Baye, Phys. Rev. Lett 58 (1987) 2738; J. of Phys. A20 (1987) 5529.

[2] S. Ali and A. R. Bodmer, Nucl. Phys. 80 (1966) 99.

[3] B. Buck, H. Friedrich and C. Wheatley, Nucl. Phys. A275 (1977) 246.

[4] A. A. Andrianov, N. V. Borisov and M. V. Ioffe, Phys. Lett. A105 (1984) 19; C. V. Sukumar, J. of Phys. A18 (1985) 2917, 2937.

[5] D. Baye and G. Reidemeister, Nucl. Phys. A258 (1976) 157; F. Michel and G. Reidemeister, J. of. Phys. G11 (1985) 835; H. G. Bohlen et al., Z. Phys. A308 (1982) 121; Y. Kondo et al., Phys. Lett. B227 (1989) 310.

[6] D. Baye, P. Descouvemont and M. Kruglanski Nucl. Phys. A550 (1992) 250; Q. K. K. Liu, Nucl. Phys. A550 (1992) 263.

[7] W. Van Dijk, M. W. Kermode, S. A. Moszkowski, (1994) preprint; D. Ridikas, J. S. Vaagen, and J. M. Bang, Nucl. Phys. A609 (1996) 21.

[8] E. Witten, Nucl. Phys. B185 (1981) 513.

[9] L. Infeld and T. T. Hull, Rev. Mod. Phys. 23 (1951) 21.

[10] For a recent review see F. Cooper, A. Khare and U. Sukhatme, Phys. Rep. 251 (1995) 267.

[11] L. Gendenshtein, JETP Lett. 38, (1983) 356.

[12] C. V. Sukumar, J. Phys. A18, (1985) L57.

[13] F. Michel and G. Reidemeister, Z. Phys. A329, (1988) 385.

[14] V. I. Kukulin et al., Sov. J. Nucl. Phys. 43, (1986) 355 ; Phys. Lett. 135 (1984) 20.

[15] R. Dutt, A. Khare and U. P. Sukhatme, Am. J. Phys. 56 (1988) 163.

[16] L. Landau and E. Lifshitz, Quantum mechanics (Pergamon, New York, 1977).

[17] R. V. Reid, Ann. Phys. (N.Y.) 50, (1968) 411.

[18] M. V. Zhukov et al., Phys. Rep. 231 (1993) 151; P. G. Hansen et al., Ann. Rev. Nucl. Part. Science 45 (1995) 591. 
[19] J. S. Winfield et al., J. Phys. G25 (1999) 755.

[20] H. Lenske and G. Schrieder, Eur. Phys. J. A, 2 (1998) 41; N. K. Timofeyuk and R. C. Johnson, Phys. Rev. C59 (1999) 1545; M. Yilmaz and B.Gönül, Gaziantep Univ. Report NPG-99/11, submitted to Few-Body Syst.(Ms$1 / 1)$.

[21] R. C. Johnson and P. J. R Soper, Phys. Rev. C1 (1970) 976.

[22] H. Amakawa, N. Austern, and C. M. Vincent, Phys. Rev. C29 (1984) 699; E. J. Stephenson et al., Phy. Rev. C42 (1990) 2562.

[23] M. Igarashi, M. Toyoma, and N. Kishida, Computer program TWOFNR (Surrey University version).

[24] F. D Bechetti and G. W. Greenlees, Phys. Rev. 182 (1969) 1190.

[25] E. Garrido, D. V. Federov and A. S. Jensen, Nucl. Phys. A650 (1999) 247. 


\section{Figure Captions}

Fig. 1-a. Superdeep potential $V_{1}(r)$ (solid line) for the deuteron system and its supersymmetric partners $V_{2}(r)$ (non-PEP, dotted line) and $V_{3}(r)$ (PEP, dashed line) as a function of radius $r$.

Fig. 1-b. Comparison of the $n-p$ central potential of the superdeep sechsquared $V_{1}(r)$ (solid line) potential and its PEP potential $V_{3}(r)$ (dashed line) with the central Reid Soft Core (dotted line) interaction.

Fig. 2. The first two eigenstates, $n=0$ for the ground state (solid line) and $n=1$ for the first excited state (dotted line) of the original hamiltonian with the superdeep two-parameter potential for the deuteron. The wave function illustrated by dashed line represents the ground state of the SUSY PEP, $V_{2}(r)$.

Fig. 3. The same as Fig.1-a, but calculations have been carried out for the ${ }^{11} \mathrm{Be}$ system.

Fig. 4. The ${ }^{11} B$ e ground-state radial wave functions $\Psi_{1}^{(n=1)}(\tilde{A}, \beta, r)$ (solid curve), $\Psi_{2}^{(n=0)}(\tilde{A}, \beta, r)$ (dotted curve) and $\Psi_{3}^{(n=0)}(\tilde{A}, \beta, r)$ (dashed curve) all normalized to one.

Fig. 5. Calculated differential cross section angular distributions within the adiabatic model for the ${ }^{11} \mathrm{Be}(p, d){ }^{10} \mathrm{Be}$ (g.s.) reaction at $35 \mathrm{MeV}$ using the deep and shallow (PEP) two-body potential descriptions for the weakly bound deuteron and ${ }^{11} \mathrm{Be}$ nuclei. 